\title{
Genetic Indices Relationship to Hyperglycemia-associated Biomarkers: Consistency with miRNA Expression in Egyptian Children with T1DM
}

\author{
(D) Naglaa Fathy Barseem¹, (D) Marwa Mohamed Mahasab2, (D Ibrahem Fathy Zaed33, (D) Aya Eldesoky A. Said33, \\ (D) Eman Masoud Abd El Gayed4
}

\author{
${ }_{1}^{1}$ Menoufia University Faculty of Medicine, Department of Pediatric, Unit of Genetic and Endocrinology, Shebein Elkom, Egypt \\ 2 Menoufia University Faculty of Medicine, Department of Family Medicine, Shebein Elkom, Egypt \\ ${ }^{3}$ Menoufia University Faculty of Sciences, Department of Chemistry, Shebein Elkom, Egypt \\ ${ }^{4}$ Menoufia University Faculty of Medicine, Department of Medical Biochemistry and Molecular Biology, Shebein Elkom, Egypt
}

What is already known on this topic?

MicroRNAs (miRNAs) are oligonucleotide sequences, some of which are known to exert a specific biological function.

What this study adds?

New evidence about miRNA expression patterns in type 1 diabetes mellitus (T1DM). Gives insights into the criteria for biomarkers to be used in T1DM. Some miRNA molecules appear to be highly predictive for disease-associated glycemic derangement, depending on the reliability of the used methodology.

\begin{abstract}
Objective: Micro RNAs (miRNAs) are gaining acceptance as novel biomarkers for the autoimmune disorders. However, miRNA profiles have not been investigated in individuals at risk of or diagnosed with type 1 diabetes mellitus (T1DM). To study the expression pattern of miRNAs in plasma obtained from patients with T1DM and compare with matched healthy controls

Methods: Equal numbers of patients with T1DM (90) and healthy-matched control children (90) were assessed for the expression profile of plasma miRNAs including miRNA-101-5p, miRNA-146-5p, miRNA-21-5p, miRNA-375, miRNA-126, and Let7a-5p using reverse transcriptase polymerase chain reaction methodology and quantitative real-time testing.

Results: Analysis showed that miRNA-101, miRNA-21 and miRNA-375 were highly expressed, whereas, miRNA-146-5p, miRNA-126, and miRNA-Let7a-5p showed significantly low levels of expression in T1DM patients compared to controls $(p<0.05)$. In addition, miRNA-101 and miRNA-1 46 correlated with age at diagnosis of T1DM and disease duration, respectively. Furthermore, multivariate analysis showed that miRNA-126 and Let7a-5p had a significant negative correlation with mean hemoglobin A1c (HbA1c) values.

Conclusion: Dysregulation of the six miRNAs analyzed suggested a possible role as biomarkers in T1DM. miRNA-101 was correlated with age at diagnosis while miRNA-146 correlated with disease duration. Two further miRNAs correlated with the existing biomarker, HbA1c.
\end{abstract}

Keywords: Type 1 diabetes, miRNAs, plasma, q RT-PCR, gene expression

\section{Introduction}

Type 1 diabetes mellitus (T1DM) is characterized by autoimmune destruction of pancreatic beta-cells by autoimmune mechanisms (1). When beta-cell destruction exceeds $80-90 \%$ by the infiltrating immune system, the disease can be diagnosed. The development of T1DM is slow, with a long latent phase during which it is possible to discover and treat individuals at risk $(2,3)$.

It is estimated that about 80,000 children may develop T1DM annually. 
Complications related to vascular pathology associated with T1DM have a major impact on quality of life, morbidity, and mortality rates, posing an enormous burden on healthcare systems worldwide. Diabetic nephropathy is a major cause of end-stage renal disease (ESRD) and T1DM also increases the risk of cardiovascular diseases. In addition, severe diabetic retinopathy may result in blindness in adult patients. Thus, the identification of novel targets for improved treatment options and innovative, non-invasive biomarkers are urgently needed to enhance risk prediction, early diagnosis, and prognostic assessment (4).

Short (22 nucleotides), non-coding microRNA (miRNA) molecules have been shown to be important agents that regulate the pattern of organic phenomenon underlying disease pathogenic mechanisms in a post-transcriptional manner (5). Generally, miRNAs exert their respective function through binding with the 3' untranslated regions of their specific genes, leading to translational inhibition or direct degradation of the targeted mRNA with a resultant decrease in protein expression $(5,6)$. Observed alterations in miRNA expression have been closely associated with many human inflammatory and autoimmune disorders $(7,8)$. The estimated regulatory control of miRNAs of more than $60 \%$ of the protein-coding genes had consequently been linked to many diseases, including cancer, endocrine disorders, and autoimmune diseases, including T1DM (9). MiRNA-specific profiles were observed in peripheral blood mononuclear cells or serum from T1DM patients and these important molecules seem to modulate mRNA expressions of the major T1DM autoantigens (10). Previously, it was reported that miRNAs, especially those expressed in human pancreatic islets, including miRNA-375 and miRNA-376, may be involved in the regulation of beta-cell pancreatic function (11). Later, a number of miRNAs were discovered to be regulatory factors for beta-cell pancreatic function (12).

We selected these beta-cell associated miRNAs, miR-101, miR-21 and-375, miR-146-5p, miR-126, and miR-Let7a-5p, as they are considered good indicators of beta-cell pancreatic function and diabetic state. The aim of the present study was to investigate the variable pattern of miRNA expression profiles in plasma obtained from patients with T1DM and matched control subjects through quantitative real-time (RT) polymerase chain reaction (PCR).

\section{Methods}

This case-control study was prospectively conducted in children with T1DM having variable disease duration and variable degrees of glycemic control, who were diagnosed according to ADA criteria (group 1) (1). A group of apparently healthy, age- and sex-matched children served as controls (group 2). All subjects were enrolled from the Pediatric Department in collaboration with the Department of Medical Biochemistry and Molecular Biology, Faculty of Medicine, Menoufia University Hospitals, Egypt. Demographic data, anthropometric measurements, treatment regimens, and other clinically important parameters were extracted from medical sheet records.

Cases that were suspected of being diagnosed as maturity onset diabetes of youth, type 2 diabetes, or secondary diabetes mellitus, or with evidence of chronic systemic/ rheumatic diseases, inflammatory disorders, and recent febrile illness, or on long-term steroid therapy, were excluded from the study.

Upon approval of the study protocol from the Ethical Committee of Menoufia University, according to the Helsinki II Declaration criteria, written informed consent was obtained from all participants.

Following complete history taking and thorough clinical examination, all studied subjects underwent sampling of 7-10 mL whole blood after an overnight fast of at least 12 hours via sterile technique. One $\mathrm{mL}$ of blood was transferred into a sodium fluoride tube and another sample of blood was obtained after 2 hours for an enzymatic colorimetric determination of blood glucose, using a commercially available kit, (Spinreact, Spain) (13).

Another $4 \mathrm{~mL}$ of blood was transferred into two EDTA tubes: one $2 \mathrm{~mL}$ sample was used for quantitative colorimetric determination of glycated hemoglobin expressed as a percentage of the total hemoglobin by the use of (Teco Diagnostics, USA) (14) and hemoglobin A1c (HbA1c) values of $\geq 6.5 \%$ was accepted as the limit for diagnosing T1 DM (1).

For molecular analysis, $2 \mathrm{~mL}$ of blood was transferred into the other EDTA tube and centrifuged for ten minutes at 4000 rpm. The clear supernatant was separated and kept frozen at $-80^{\circ} \mathrm{C}$ until further processing. Determination of miRNA levels was performed through a process to obtain cDNA via reverse transcription of previously isolated RNA, together with the measurement of miRNA levels using specific primer sets after being referenced against endogenous control U6B. These steps were as follows.

\section{RNA Isolation}

A total RNA, including miRNA molecules was extracted from plasma using Qiagen ${ }^{\mathrm{TM}}$ RNA Blood Mini Kit (Qiagen, Applied Biosystems, USA) according to the manufacturer's instructions. 


\section{Real-time PCR}

The Qiagen ${ }^{\circledR}$ miScript $\quad$ II $\quad$ RT $\quad$ Kit (Qiagen, Applied Biosystems, USA) was used to reverse transcribe RNA. Then, complementary DNA (CDNA) was assayed with the universal SYBR Green Master Mix (QuantiTect SYBR Green PCR Kit, Qiagen).

The preparation of the RT Master Mix was made as follows. $4 \mu \mathrm{L} 5 \times$ miScript HiSpec Buffer, $2 \mu \mathrm{L} 10 \times$ miScript Nuclease Mix, $2 \mu \mathrm{L}$ RNase-free water, $2 \mu \mathrm{L}$ miScript reverse transcriptase Mix, then a $10 \mu \mathrm{L}$ template RNA to achieve a total reaction volume of $20 \mu \mathrm{L}$. Reverse transcription was carried out at $37{ }^{\circ} \mathrm{C}$ for 60 minutes and $95^{\circ} \mathrm{C}$ for 5 minutes on an Applied Biosystems 2720 thermal cycler (Bioline, USA). Diluted CDNA was the template for the second step, RT-PCR using Qiagen-produced SYBR Green miScript kit. The addition of universal primers was based on mRNA sequences delivered from the miR-database for miRNA-101-5p, miRNA-146a-5p, miRNA-375, miRNA21-5p, miRNA-126, and miRNA Let $7 \mathrm{a}-5 \mathrm{p}$, as shown in Table 1. Each reaction for RT-PCR was finalized to $25 \mu \mathrm{L}$ volume, as follows: $12.5 \mu \mathrm{L} 2 \mathrm{x}$ QuantiTect SYBR Green PCR Master Mix, $2.5 \mu \mathrm{L}$ 10x miScript specific Primer, $2.5 \mu \mathrm{L} \mathrm{10x}$ miScript primer assay, $4 \mu \mathrm{L}$ Template $\mathrm{c}$ DNA and $3.5 \mu \mathrm{L}$ RNase-free water. The mixture was incubated at $95{ }^{\circ} \mathrm{C}$ for 15 min (as initial denaturation), then denaturation at 94 ${ }^{\circ} \mathrm{C}$ for 15 seconds duration, annealing for 30 seconds at a temperature of $55{ }^{\circ} \mathrm{C}$ and final extension for 30 seconds adjusted to $70{ }^{\circ} \mathrm{C}$, for 60 cycles. Amplification of small RNA RNU6B was carried out with each sample as an endogenous control. Data analysis was performed by the RT cycler inbuilt software (Applied Biosystems ${ }^{\circledR} 7500$ Software version 2.0.1 thermal cycler, Applied Biosystems, Foster City, CA, USA).

\section{Validation of the Quantified miRNAs}

Subsequently, relative quantification of gene expression was performed using the comparative $\Delta \Delta \mathrm{Ct}$ method, in which the amount of targeted miRNAs were normalized to RNU6B as an endogenous reference for both patients and controls.
It should be noted that the target miRNAs were selected because of evidence from available databases and literature that showed the association of these molecules to pathways involved in T1DM development in humans $(2,9,15)$.

\section{Statistical Analysis}

Statistical Package for the Social Sciences (SPSS), version 20 (SPSS Inc., Chicago, USA) was used for data analysis. For nonparametric data, median and range values were used. A chisquare test was used to examine the relationship between qualitative variables. For quantitative data, a comparison between two groups was done using either Student's t-test or Mann-Whitney test (non-parametric t-test), as appropriate. The correlation between numerical variables was assessed using Spearman's correlation method. For the determination of T1DM risk, multivariate logistic regression analysis was additionally used, aided by the calculation of Odds ratio (OR) and $95 \%$ confidence Interval (CI). A p-value less than 0.05 was considered significant.

\section{Results}

In this study, a total of 180 children were enrolled, including 90 (46 males and 44 females) children with T1DM (group 1), and 90 (55 males and 35 females) control subjects (group 2) who were age and sex matched. The mean age of patients and controls was $10.93 \pm 4.51$ and $10.15 \pm 2.56$ years, respectively. Demographic and clinical data of the studied groups are shown in Table 2.

Unsurprisingly, results of laboratory investigations for the T1DM children including fasting blood sugar, 2-hour postprandial blood sugar (2-hPP) and mean HbA1c\% were significantly different to the healthy controls $(p<0.001$ for all). All of the newly diagnosed cases with T1DM disease duration of 6-12 months were diagnosed because of being positive for anti-insulin autoantibodies (IAAs), where the levels ranged from 10-130 mIU/L with a mean value of $10.42 \pm 19.59$ compared to healthy controls with IAA range of 0-7.0 mIU/L and mean value of $2.08 \pm 2.36(p=0.002)$.

Table 1. Primers used for the quantitative reverse transcriptase-polymerase chain reaction assay for micro-RNA determination
in all samples
\begin{tabular}{llll} 
Mature miRNA symbol & Accession No. & Sequence & Catalogue No. \\
\hline miR 101-5p & MIMAT0004513 & 5-CAGUUAUCACAGUGCUGAUGCU-3 & MS00008379 \\
miR 146 a-5p & MIMAT0000449 & 5-UGAGAACUGAAUUCCAUGGGUU-3 & MS00003535 \\
miR 21 a-5p & MIMAT0004494 & 5-CAACACCAGUCGAUGGGCUGU-3 & MS000009086 \\
miR-375 & MIMAT00000728 & 5-UUUGUUCGUUCGGCUCCGUGA-3 & MS000031829 \\
miR 126 a-3p & MIMAT0000445 & 5-UCGUACCGUGAGUAAUAAUGCG-3 & MS00003430 \\
Let 7 a-5p & MIMAT0000062 & 5-UGAGGUAGUAGGUUGUACAGUU-3 & MS00031220 \\
RNU6B & Internal control & 5-CGCAAGGATGACACGCAAATTCGTGAAGCGTTCCATATTTTT-3 & MS00033740 \\
\hline
\end{tabular}


Comparative results regarding the levels of miRNAs studied in both groups revealed that miRNA-101-5p, miRNA21-5p, miRNA-375 were present in significantly higher concentration in patients with T1DM $(p<0.05)$, whereas
miRNA146-5p, miRNA-126, and miRNA Let 7a-5p had significantly lower plasma levels in T1DM $(p<0.05)$ (Table 3). One of the remarkable findings in this study were significant negative correlations between miRNA-101-

Table 2. Comparison between the two studied group as regarding to demographic, clinical and laboratory data

\begin{tabular}{|c|c|c|c|c|c|c|}
\hline & \multicolumn{2}{|c|}{ T1DM $(n=90)$} & \multicolumn{2}{|c|}{ Control $(n=90)$} & \multirow[t]{2}{*}{ Test of sig. } & \multirow[t]{2}{*}{$\mathrm{p}$} \\
\hline & No. & $\%$ & No. & $\%$ & & \\
\hline \multicolumn{7}{|l|}{ Sex } \\
\hline Male & 46 & 51.1 & 55 & 61.1 & \multirow[t]{2}{*}{$\chi^{2}=1.827$} & \multirow[t]{2}{*}{0.176} \\
\hline Female & 44 & 48.9 & 35 & 38.9 & & \\
\hline \multicolumn{7}{|l|}{ Consanguinity } \\
\hline Positive & 48 & 48 & 65 & 65 & \multirow[t]{2}{*}{2.9} & \multirow[t]{2}{*}{0.006} \\
\hline Negative & 52 & 52 & 35 & 35 & & \\
\hline \multicolumn{7}{|l|}{ Family history } \\
\hline Positive & 21 & 21 & 0 & 0 & & \multirow{2}{*}{$<0.001 *$} \\
\hline Negative & 79 & 79 & 100 & 100 & $20.1^{*}$ & \\
\hline \multicolumn{7}{|l|}{ Age (years) } \\
\hline Mean \pm SD & \multicolumn{2}{|c|}{$10.93 \pm 4.51$} & \multicolumn{2}{|c|}{$10.15 \pm 2.56$} & $\mathrm{t}=1.434$ & 0.154 \\
\hline \multicolumn{7}{|c|}{ Systolic blood pressure (mm/Hg) } \\
\hline Mean \pm SD & \multicolumn{2}{|c|}{$110.4 \pm 6.10$} & \multicolumn{2}{|c|}{$107.0 \pm 4.50$} & $\mathrm{t}=3.199^{\circ}$ & $0.002^{*}$ \\
\hline \multicolumn{7}{|c|}{ Diastolic blood pressure $(\mathrm{mm} / \mathrm{Hg})$} \\
\hline Mean \pm SD & \multicolumn{2}{|c|}{$67.78 \pm 5.95$} & \multicolumn{2}{|c|}{$67.0 \pm 6.44$} & $\mathrm{t}=0.841$ & 0.401 \\
\hline \multicolumn{7}{|l|}{ BMI $\left(\mathrm{kg} / \mathrm{m}^{2}\right)$} \\
\hline Mean (SD) & 19.6 & & 20.2 & & $t=0.91$ & 0.32 \\
\hline Range & $13-2$ & & 13.3 & & & \\
\hline Age of onset & & & & & & \\
\hline Mean \pm SD & 6.93 & & & & & \\
\hline Median (IQR) & 7.0 & & & & & \\
\hline Duration of illness & & & & & & \\
\hline Mean \pm SD & 4.41 & & & & & \\
\hline Median (IQR) & 4.42 & & & & & \\
\hline Presentation & & & & & & \\
\hline DKA & 30 & 33.3 & & & & \\
\hline Classic symptoms & 60 & 66.7 & & & & \\
\hline HbA1c\% & & & & & & \\
\hline Mean \pm SD & 9.03 & & 5.94 & & $t=14.165^{*}$ & $<0.001 *$ \\
\hline Median (IQR) & 9.0 & & 5.95 & & & \\
\hline FBS (mg/dL) & & & & & & \\
\hline Min-max & 105. & & 75.0 & & & \\
\hline Mean \pm SD & 210 & & 91.0 & & $\mathrm{t}=23.985^{*}$ & $<0.001^{*}$ \\
\hline Median (IQR) & 200 & $0-250.0)$ & 91.0 & $3.0)$ & & \\
\hline $2 \mathrm{hr}$ PP (mg/dL) & & & & & & \\
\hline Min-max & 130. & & 140 & & & \\
\hline Mean \pm SD & 257. & & 154 & & $t=23.156^{*}$ & $<0.001 *$ \\
\hline Median (IQR) & 260. & $0-300.0)$ & 155 & $-165.0)$ & & \\
\hline Micro albuminuria & & & & & & \\
\hline No & 82 & 91.1 & 91 & 100.0 & 7 & $\mathrm{FE} \mathrm{P}-0007^{*}$ \\
\hline Yes & 8 & 8.9 & 0 & 0.0 & $\chi^{2}=8.372$ & $p=0.00 \%$ \\
\hline Min-max & 206. & & - & & & \\
\hline Mean \pm SD & 265. & 19 & - & & - & - \\
\hline Median (IQR) & 231. & $(-316.0)$ & - & & & \\
\hline
\end{tabular}


$5 p$ and the age of T1DM onset $(r=-0.264, p=0.015)$ and with the duration of T1DM $(r=-0.162, p=0.02)$ while MiR146 correlated positively with disease duration $(r=0.239$, $p=0.023)$. Furthermore, miRNA-126 $(r=-0.214, p=0.042)$ and miRNA-Let7a-5p $(r=-0.216, \quad p=0.043) \quad$ correlated negatively with mean HbA1c levels (Table 4).
Results of multivariate logistic regression analysis for T1DM risk are shown in Table 5. miRNA-126 and miRNA-Let7a$5 \mathrm{p}$ markers remained highly significant after adjustment for age, sex and mean $\mathrm{HbA} 1 \mathrm{c}$ levels with OR $(95 \% \mathrm{CI})$ of 0.016 (0.0-0.544), $p=0.021$ for miRNA-126 and 1.808 (1.0063.249) $\mathrm{p}=0.048$ for miRNA-Let7a-5p.

\begin{tabular}{|c|c|c|c|c|c|}
\hline miRNA expressions & T1DM $(n=90)$ & Control $(n=90)$ & $\mathrm{U}$ & & $\mathrm{p}$ \\
\hline \multicolumn{6}{|l|}{ miRNA-101-5p } \\
\hline Min-max & $0.0-1640.55$ & $0.09-1.66$ & 3652.0 & & $0.028^{*}$ \\
\hline Mean \pm SD & $65.33 \pm 270.39$ & $0.39 \pm 0.14$ & & & \\
\hline Median (IQR) & $0.60(0.08-1.95)$ & $0.26(0.10-0.35)$ & & & \\
\hline \multicolumn{6}{|l|}{ miRNA-146a-5b } \\
\hline Min-max & $0.0-328.49$ & $0.0-1.21$ & $2646.0^{*}$ & & $<0.001$ * \\
\hline Mean \pm SD & $7.89 \pm 48.63$ & $0.63 \pm 0.44$ & & & \\
\hline Median (IQR) & $0.16(0.02-0.43)$ & $0.77(0.0-0.88)$ & & & \\
\hline \multicolumn{6}{|l|}{ miRNA-375a-3p } \\
\hline Min-max & $0.0-2127.09$ & $0.07-2.93$ & $1908.0 *$ & & $<0.001$ * \\
\hline Mean \pm SD & $70.36 \pm 332.53$ & $0.95 \pm 1.02$ & & & \\
\hline Median (IQR) & $2.10(1.08-3.11)$ & $0.53(0.09-1.05)$ & & & \\
\hline \multicolumn{6}{|l|}{ miRNA-21-3p } \\
\hline Min-max & $0.0-89.39$ & $0.0-0.06$ & $2682.0^{*}$ & & $<0.001$ * \\
\hline Mean \pm SD & $2.27 \pm 13.21$ & $0.03 \pm 0.02$ & & & \\
\hline Median (IQR) & $0.08(0.0-0.48)$ & $0.02(0.0-0.05)$ & & & \\
\hline \multicolumn{6}{|l|}{ miRNA-126 } \\
\hline Min-max & $0.00-0.62$ & $0.00-152.32$ & $1569.50 *$ & $<0.001^{*}$ & \\
\hline Mean \pm SD & $0.19 \pm 0.15$ & $4.50 \pm 22.39$ & & & \\
\hline Median (IQR) & $0.15(0.07-0.31)$ & $0.69(0.26-1.61)$ & & & \\
\hline \multicolumn{6}{|l|}{ miRNA Let $7 a-5 p$} \\
\hline Min-max & $0.0-35.80$ & $0.0-1.92$ & $2356.50^{*}$ & $<0.001^{*}$ & \\
\hline Mean \pm SD & $0.94 \pm 5.21$ & $0.50 \pm 0.51$ & & & \\
\hline Median (IQR) & $0.09(0.02-0.23)$ & $0.12(0.10-1.02)$ & & & \\
\hline
\end{tabular}

\begin{tabular}{|c|c|c|c|c|c|c|c|}
\hline & & miRNA-101 -5p & miRNA-146a-5b & miRNA-375a-5p & miRNA-21 -3p & miRNA-126 & miRNA Let $7 a-5 p$ \\
\hline \multirow[t]{2}{*}{ Duration of illness } & $r_{s}$ & -0.162 & 0.239 & 0.095 & -0.151 & -0.167 & -0.034 \\
\hline & $\mathrm{P}$ & $0.02 *$ & $0.023^{*}$ & 0.386 & 0.167 & 0.128 & 0.756 \\
\hline \multirow[t]{2}{*}{ Age of onset } & $r_{s}$ & -0.264 & 0.149 & -0.008 & $0.211 *$ & 0.089 & -0.062 \\
\hline & $\mathrm{P}$ & $0.015^{*}$ & 0.160 & 0.943 & $0.046^{*}$ & 0.402 & 0.563 \\
\hline \multirow[t]{2}{*}{$\mathrm{HbA1c}$} & $r_{s}$ & 0.090 & 0.183 & 0.173 & 0.054 & -0.214 & -0.216 \\
\hline & $\mathrm{P}$ & 0.413 & 0.162 & 0.201 & 0.610 & $0.042^{*}$ & $0.043 *$ \\
\hline \multirow[t]{2}{*}{ FBS } & $r_{s}$ & -0.194 & 0.176 & -0.147 & 0.166 & -0.032 & 0.241 \\
\hline & $\mathrm{P}$ & 0.067 & 0.097 & 0.166 & 0.119 & 0.764 & 0.022 \\
\hline \multirow[t]{2}{*}{$2 \mathrm{hr} P \mathrm{PP}$} & $r_{s}$ & -0.215 & 0.134 & -0.008 & 0.096 & 0.006 & 0.123 \\
\hline & $\mathrm{P}$ & 0.042 & 0.209 & 0.939 & 0.368 & 0.954 & 0.247 \\
\hline
\end{tabular}


Correlations between some of the studied mi-RNAs and some clinical parameters of T1DM are shown in Figures 1, 2. The amplification for miRNAs expression patterns [normalized fluorescent signal $(\Delta R n)$ that was plotted against the number of cycles] is shown in Figures 3,4,5.

\section{Discussion}

The understanding of the role of miRNAs in modulating gene expression has greatly improved and these molecules are being implicated in the disease mechanisms of various genetic disorders $(16,17,18)$. In light of this, miRNAs have been proposed as biomarkers of disease pathogenesis and prognosis (19,20). This suggestion was supported by the relationship of these molecules to $60 \%$ or more of the coding genes that were thought to be associated with a number of endocrine and autoimmune diseases $(21,22,23)$.

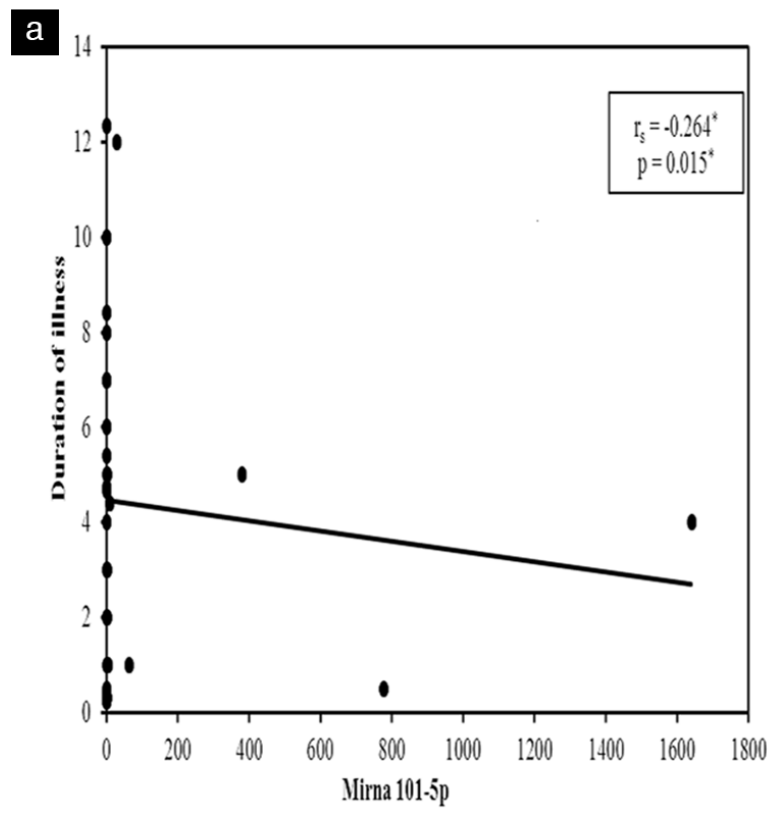

Based on the underlying autoimmune background of T1DM, it seemed reasonable to include children with recently diagnosed T1 DM who still had circulating IAA. Some studies have suggested that miRNAs may be helpful as biomarkers in the early phases of T1DM (22). Other miRNAs are closely associated with glucose homeostasis that determines the progressive pattern in at-risk individuals (22). There is recent evidence of changes in proinflammatory cytokines and autoimmune markers which is asociated with particular patterns of circulating miRNAs in children with T1DM $(24,25,26)$.

Consistent with this, we found that miRNA-146a-5p, miRNA Let-7a-5p, and miRNA-126 were down-regulated, whereas miRNA-101, miRNA-21-5p, and miRNA-375a-5p were consistently up-regulated in patients compared to controls.

As for miR-375, its abundance in pancreatic tissue has been suggested to make it a good biomarker for beta-cell mass

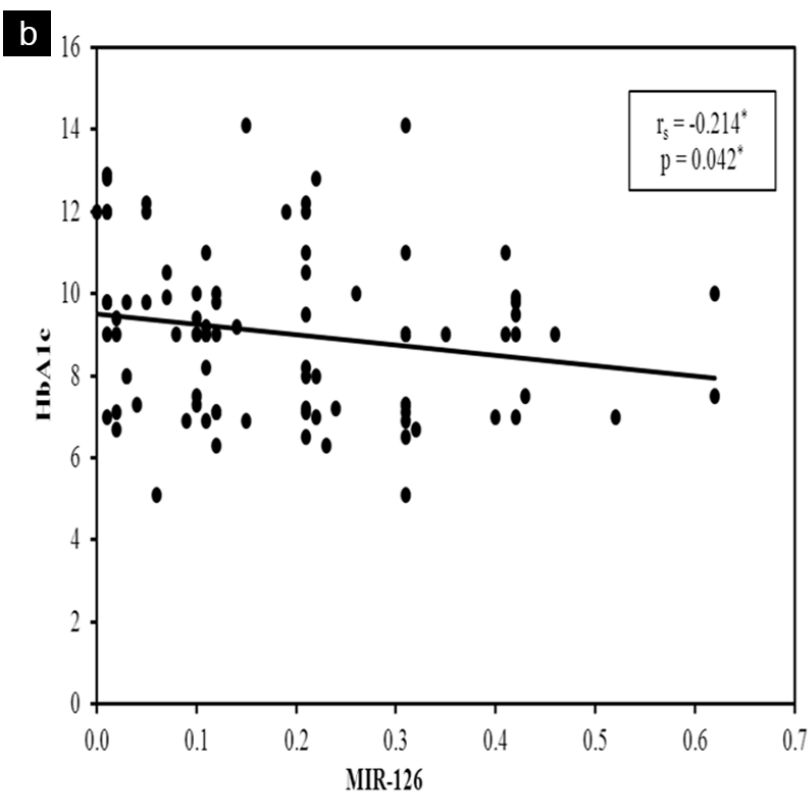

Figure 1. a) Correlation results between miRNA-101-5p with age of type 1 diabetes mellitus onset. b) Correlation results between miRNA-126 and hemoglobin A1C

Table 5. Univariate and multivariate analysis for the parameters affecting T1DM risk $(\mathrm{n}=90)$

\begin{tabular}{|c|c|c|c|c|}
\hline & \multicolumn{2}{|c|}{ Univariate } & \multicolumn{2}{|c|}{ Adjust OR } \\
\hline & $\mathrm{p}$ & COR $(95 \% \mathrm{CI})$ & $\mathrm{p}$ & $\operatorname{AOR}^{\#}(95 \% \mathrm{CI})$ \\
\hline miRNA-101 $-5 p$ & $0.009^{*}$ & $1.565(1.121-2.185)$ & 0.307 & $1.783(0.587-5.414)$ \\
\hline miRNA Let $7 a-5 p$ & $<0.001^{*}$ & $1.845(1.370-2.483)$ & $0.048 *$ & $1.808(1.006-3.249)$ \\
\hline miRNA-21-5p & $<0.001^{*}$ & $11.62(3.63 .69-71.85)$ & $0.026^{*}$ & $7.180(2.554-20.187)$ \\
\hline
\end{tabular}

*: Statistically significant at $p \leq 0.05$.

OR: Odds ratio, CI: confidence interval, AOR*: adjust OR by family history and $\mathrm{HbA} 1 \mathrm{c}, \mathrm{HbA} 1 \mathrm{c}$ : hemoglobin A1 c, miRNA: micro RNA 
and changes in beta-cell function (27). We found that the level of miRNA-375 in the plasma of patients with T1DM was significantly increased. However, in our cohort there was no correlation between miRNA-375 and HbA1c. In contrast, Marchand et al (28) reported dysregulated miRNA-375 levels in the blood of newly diagnosed children with T1DM when quantified to high levels in human islet tissue and conferred as a hallmark in the etiology of T1DM. These same authors

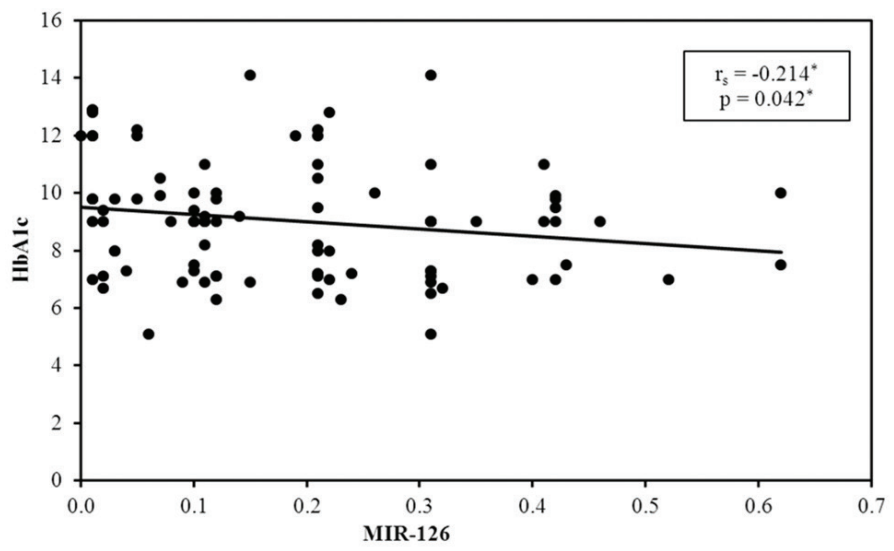

Figure 2. Amplification plot for miRNA-21-5p expression pattern [normalized fluorescent signal $(\Delta R n)$ plotted against the number of the cycle]

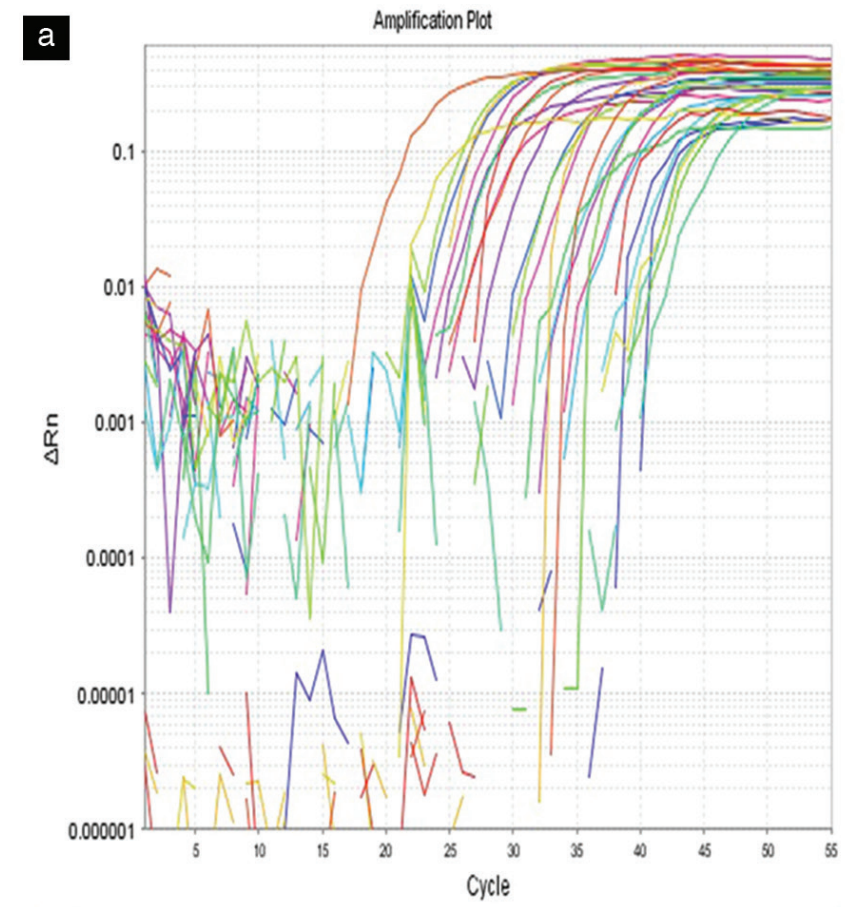

$\square$ A $\square$ B $\square$ C $\square D \square E \square F \square G \square H$ suggested miRNA-375 may be a marker of the early phases of the disease.

We found that miRNA-21-5p was present at significantly higher concentrations in T1DM patients than in the controls. Pan et al (29) investigated this same miRNA in terms of the effect of enteroviral infection on miRNA-21-5p expression and subsequent contribution to T1DM. Earlier studies have shown that has-miRNA-21-5p was highly expressed in the plasma of T1DM patients in comparison to controls (30). Ongoing research has linked miRNA-21$5 p$ to inflammatory cytokines (31). Furthermore, it was suggested that miRNA-21 overexpression was believed to influence the Bax group/apoptotic signaling pathway, and hence may be involved in pancreatic beta-cell death $(32,33)$. This could suggest a new target for T1 DM therapy.

Another up-regulated miRNA in our study was miRNA-101$5 p$, which has been associated with reduction of insulin secretion and beta-cell mass although it is known to play a role in cytokine release regulation and altered signaling of STAT3, HGF/C-Met, and Ephrin receptor/pathway mechanisms. We found a significant association of miRNA101-5p and IAA positive cases in recent onset T1DM. Interestingly, Santos et al (34) reported that the expression

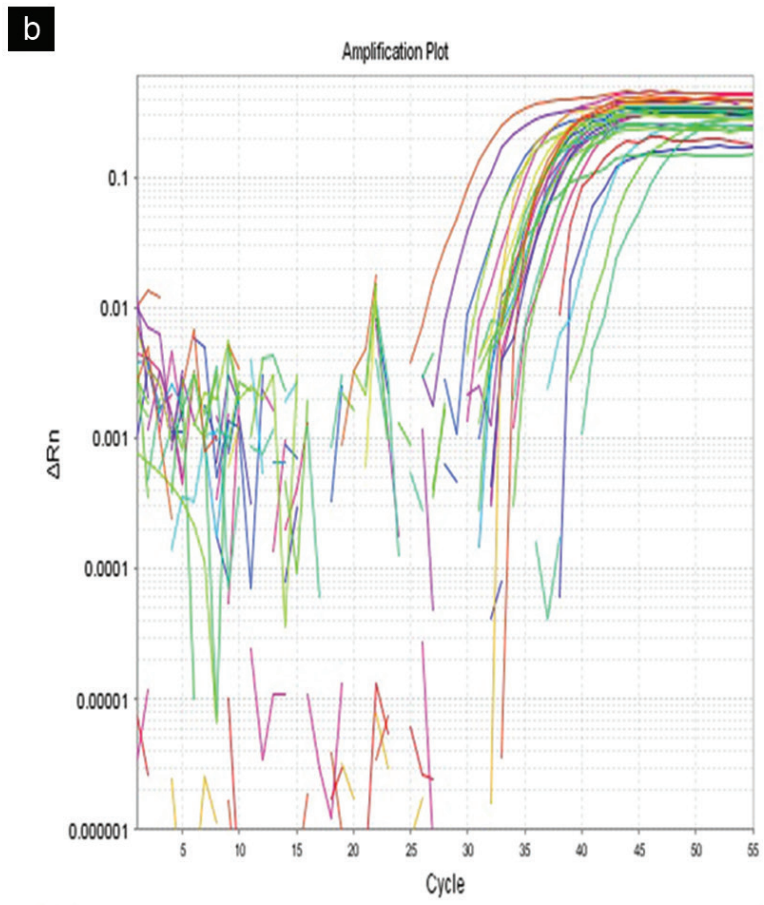

$\square$ L

Figure 3. a) Amplification for miRNA-101-5p expression [normalized fluorescent signal $(\Delta R n)$ plotted against the number of the cycle]. b) Amplification for miRNA-146a-5p expression [normalized fluorescent signal ( $\Delta \mathrm{Rn}$ ) plotted against the number of the cycle] 

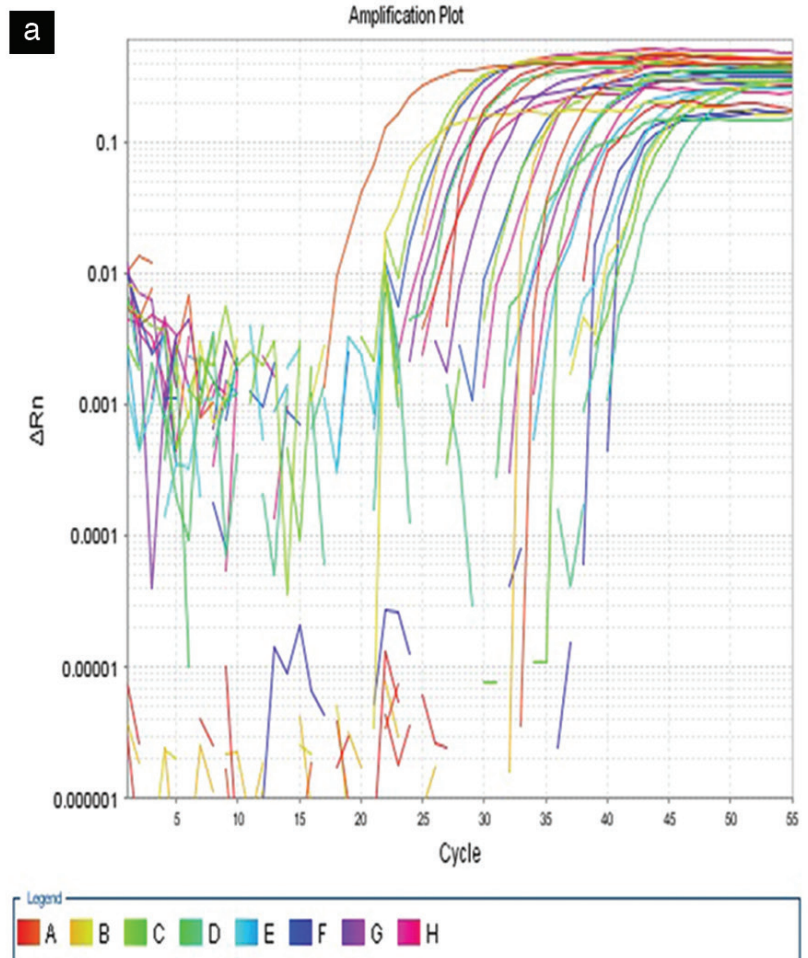

b

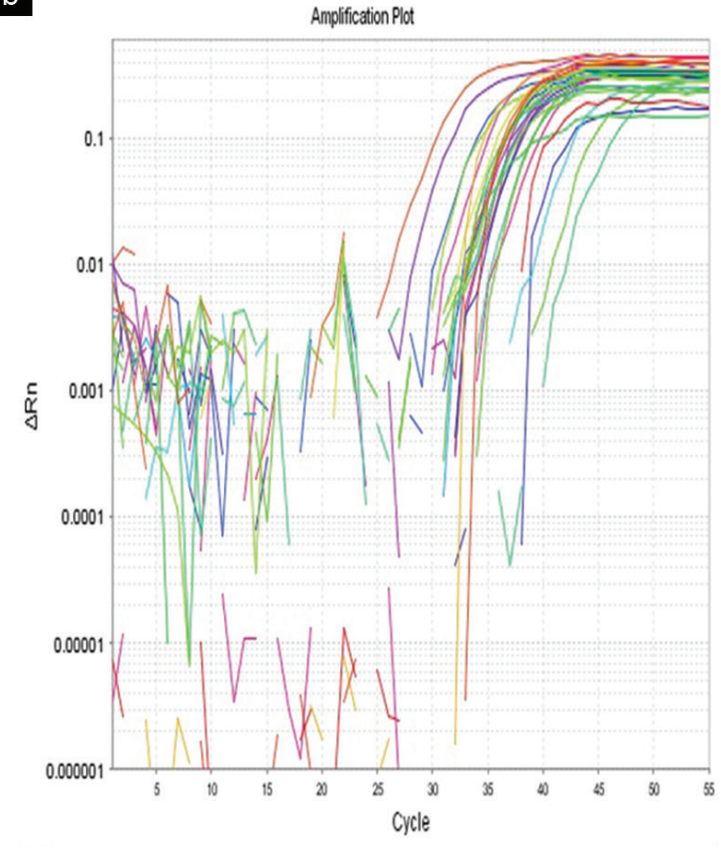

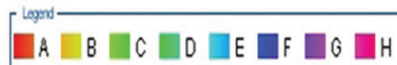

Figure 4. a) Amplification for miRNA-375 expression [normalized fluorescent signal $(\Delta R n)$ plotted against the number of the cycle]. b) Amplification plot of miRNA Let $7 \mathrm{a}-5 \mathrm{p}$ expression [normalized fluorescent signal $(\Delta \mathrm{Rn})$ against the number of the cycle]

a

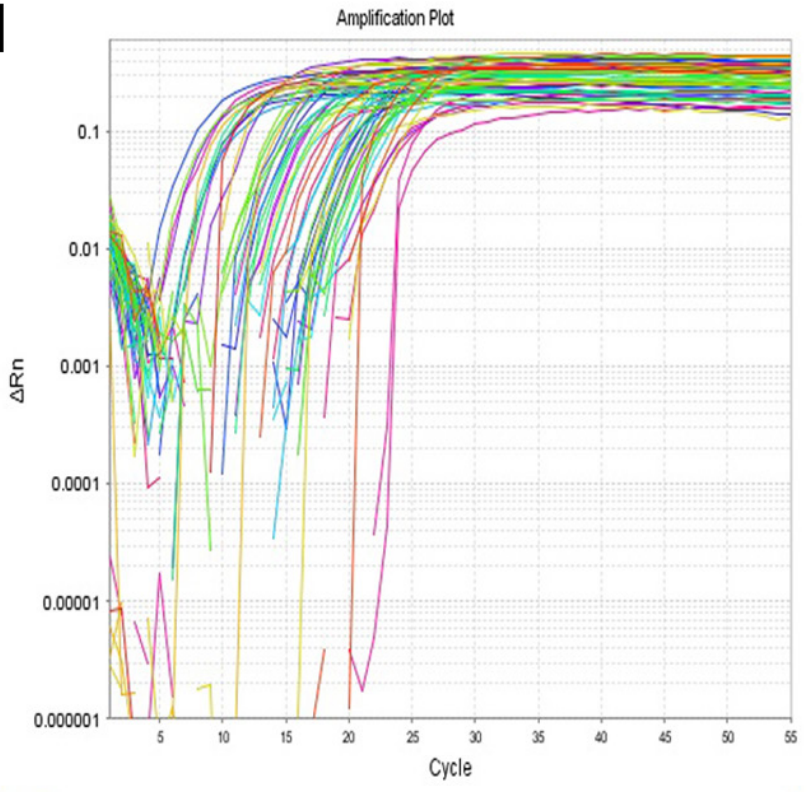

$\square \mathrm{A} \square \mathrm{B} \square \mathrm{C} \square \mathrm{D} \square \mathrm{E} \square \mathrm{F} \square \mathrm{G} \square \mathrm{H}$ b

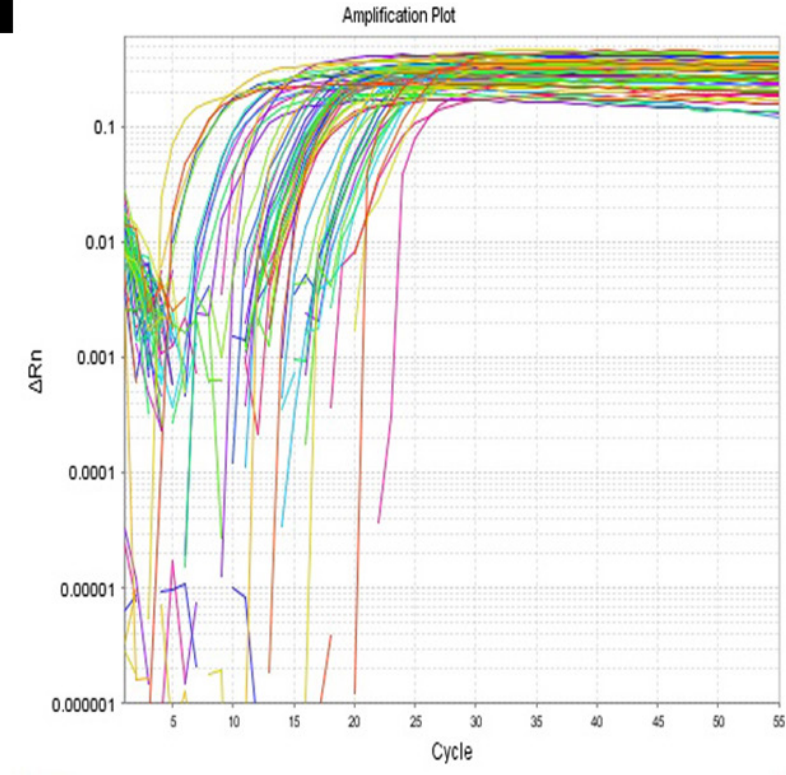

$\square \mathrm{A} \square \mathrm{B} \square \mathrm{C} \square \mathrm{D} \square \mathrm{E} \square \mathrm{F} \square \mathrm{G} \square \mathrm{H}$

Figure 5. a) Amplification of miRNA-126 gene expression [normalized fluorescent signal ( $\triangle \mathrm{Rn}$ ) against the number of the cycle]. b) Amplification of miRNA-126 gene expression [normalized fluorescent signal $(\Delta \mathrm{Rn})$ against the number of the cycle] 
of miRNA-101 was about threefold higher in patients with multiple autoantibodies levels.

miRNA Let7 a-5p was expressed at lower levels our patient group and demonstrated a statistically significant difference in comparison to that of controls. Similar results were reported by Tian et al (35) where miRNA Let7a was downregulated in both human and mouse tissue derivatives. The miRNA Let7a is known to be involved in the regulation of glucose metabolism. This molecule was found to be negatively correlated to HbA1c by Erener et al (36), which is consistent with our findings.

Assessing the level of miRNA-126 expression revealed contradictory findings. Osipova et al (30) reported lower urinary levels in patients with T1DM, with no significant difference in plasma concentration comparing patients and controls. However, Wang et al (37) found decreased plasma levels of miRNA-126 in those with chronic ESRD. We also found significantly lower plasma level of the miRNA-126 in T1DM patients in agreement with Wang et al (37) but in contrast to Osipova et al (30). It has been shown that decreased levels of miRNA-126 are associated with deranged response to vascular endothelial growth factor and endothelial dysfunction (38,39). In addition, previous reports considered miRNA-126 to be a controlling factor for various biological processes $(40,41,42)$, through linkage of decreased circulatory miRNA-126 levels to micro-vascular change, which in turn is known to influence the development of later chronic T1DM complications $(42,43)$.

The significant negative correlation between miRNA-126, mi-RNA Let7a-5p and higher mean HbA1c values suggested a significant association of the altered levels of the circulating miRNAs to hyperglycemic state (36). These findings are in agreement with the hypothesis of Akerman et al (22) who suggested that the expression of miRNAs may be of value as complementary markers in at-risk individuals with abnormal OGTT results. Satake et al (44) reported the association of dysregulated miRNAs with hyperglycemia in children with T1DM that may contribute to further development of diabetic complications.

It has been proposed that, in T1DM, miRNAs may be a cornerstone in pathogenesis and are more than merely markers of active beta-cell dysfunction (45). Previous studies reported the effect of miRNAs on pancreatic cellular biology, especially for beta-cell differentiation, insulin production, mediation of inflammation, and apoptosis (46).

There was a negative correlation between miRNA-101 with the age of onset of T1DM in our cohort. Earlier studies have suggested a greater rate of beta-cell turnover and pancreatic injury in young children with T1DM and lower levels of miRNA-101 as it functions to mediate Treg function in controlling autoimmune cellular destruction (47).

Another important miRNAs molecule that showed significant down-regulation besides being indicated in patients with recent-onset T1DM was miRNA-146 a-5p. This was evident through a lowered expression level in T1DM cases. This is of interest because miRNA-1 46-5p has been associated with genes linked to apoptotic and innate immune regulatory pathway mechanisms and, as such, warrants further investigation in T1DM (47).

\section{Study Limitations}

Small sample size was a limitation in our study so that it was not possible to reach any definite causative conclusions but merely report associations. Other limitations of our study include no calculation of sample size or power of the study. This was because published data on the link between T1DM and miRNAs are very scarce and so were not sufficient for an exact calculation of statistical sample size. Lastly, the relationship between miRNAs and the presence and/or levels of T1DM-associated autoantibodies was not investigated. Further research is necessary to overcome all of these limitations.

\section{Conclusion}

In this study there was a significant difference between plasma concentrations of some miRNAs in children with T1DM and healthy controls. In the patients with T1DM there was evident down-regulation of miRNAs-146$5 p, 126-5 p$, and Let $7 a-5 p$ molecules and up-regulation of miRNAs-101-5p, 21-5p, and 375. As miRNA-126$5 p$ and Let7a-5p miRNAs have a significant negative correlation with mean HbA1c they could be used as alternative biomarkers for hyperglycemia-associated pathophysiologic changes in T1DM and further work may reveal additional benefits of using these miRNAs compared to measuring HbA1c.

Given the known gene regulatory action of miRNAs and that we found significant changes in circulating levels in children with T1DM compared to healthy age- and sex-matched controls, there is a potential for T1DM-associated miRNAs to become useful biomarkers and even possible therapeutic targets in the future. Further larger-scale functional studies are required to investigate genetic interactions which may lead to improvement in the quality and life expectancy of children with T1DM. 


\section{Ethics}

Ethics Committee Approval: The study were approved by the Menoufia University Faculty of Medicine of Ethical Committee (approval number: 59601070, date: 09.02.2020).

Informed Consent: All subjects gave written, informed consent through their parent or guardian before enrollment in the study.

Peer-review: Externally and internally peer-reviewed.

\section{Authorship Contributions}

Surgical and Medical Practices - Concept - Design - Data Collection or Processing - Analysis or Interpretation Literature Search - Writing: All authors.

Financial Disclosure: The authors declared that this study received no financial support.

\section{References}

1. American Diabetes Association. Classification and diagnosis of diabetes. Diabetes Care 2015;38(Suppl):S8-S16.

2. Guay C, Regazzi R. Circulating microRNAs as novel biomarkers for diabetes mellitus. Nat Rev Endocrinol 2013;9:513-521. Epub 2013 Apr 30

3. Atkinson MA, Eisenbarth GS. Type 1 diabetes: new perspectives on disease pathogenesis and treatment. Lancet 2001;358:221-229.

4. Barutta F, Bellini S, Mastrocola R, Bruno G, Gruden G. MicroRNA and Microvascular Complications of Diabetes. Int $\mathrm{J}$ Endocrinol 2018;2018:6890501.

5. Kaur S, Pociot F. miRNAs regulate development and function of regulatory T-cells in recent onset islet autoimmunity in pre-Type 1 diabetes. Non-coding RNA Investig 2018;2:16.

6. Lewis BP, Burge CB, Bartel DP. Conserved seed pairing, often flanked by adenosines, indicates that thousands of human genes are microRNA targets. Cell 2005;120:15-20.

7. Sebastiani G, Spagnuolo I, Patti A, Grieco FA. MicroRNA expression fingerprint in serum of type 1 diabetic patients. Diabetologia 55:S48-S48.

8. Nielsen LB, Wang C, Sørensen K, H Bang-Berthelsen C, Hansen L, M Andersen ML, Hougaard P, Juul A, Zhang C-Y, Pociot F, B Mortensen H. Circulating levels of microRNA from children with newly diagnosed type 1 diabetes and healthy controls: evidence that miR-25 associates to residual beta-cell function and glycaemic control during disease progression. Exp Diabetes Res 2012;2012:896362.

9. Lin S, Gregory RI. MicroRNA biogenesis pathways in cancer. Nat Rev Cancer 2015;15:321-333.

10. Abuhatzira L, Xu H, Tahhan G, Boulougoura A, A Schäffer A, L Notkins A. Multiple microRNAs within the $14 q 32$ cluster target the mRNAs of major type 1 diabetes autoantigens IA-2, IA-2 $\beta$, and GAD65. FASEB J 2015;29:4374-4383. Epub 2015 Jul 6

11. Ardekani AM, Naeini MM. The Role of MicroRNAs in Human Diseases. Avicenna J Med Biotechnol 2010;2:161-179.

12. Pandey AK, Agarwal P, Kaur K, Datta M. MicroRNAs in diabetes: tiny players in big disease. Cell Physiol Biochem 2009;23:221-232. Epub 2009 May 6
13. Trinder P. Determination of Glucose in Blood Using Glucose Oxidase with an Alternative Oxygen Acceptor. Ann Clin Biochem 1969;6:24-25.

14. Gonen B, Rubenstien AH. Determination of glycohemoglobin. Diabetologia 1978;15:1-5.

15. Dweep H, Gretz N. miRWalk2.0: a comprehensive atlas of microRNAtarget interactions. Nat Methods 2015;12:697.

16. Bhatt $\mathrm{K}$, Mi QS, Dong Z. MicroRNAs in kidneys: Biogenesis, regulation, and pathophysiological roles. Am J Physiol Renal Physiol 2011;300:F602-10. Epub 2011 Jan 12

17. Butz H, Kinga N, Racz K, Patocs A. Circulating miRNAs as biomarkers for endocrine disorders. J Endocrinol Invest 2016;39:1-10. Epub 2015 May 28

18. Carrington JC, Ambros V. Role of microRNAs in plant and animal development. Science 2003;301:336-338

19. Lin S, Gregory RI. MicroRNA biogenesis pathways in cancer. Nat Rev Cancer 2015;15:321-333

20. Pauley KM, Cha S, Chan EK. MicroRNA in autoimmunity and autoimmune diseases. J Autoimmun 2009;32:189-194. Epub 2009 Mar 19

21. Zalts $\mathrm{H}$, Shomron N. The impact of microRNAs on endocrinology. Pediatr Endocrinol Rev 2011;8:354-362.

22. Akerman L, Casas R, Ludvigsson J, Tavira B, Skoglund C. Serum miRNA levels are related to glucose homeostasis and islet autoantibodies in children with high risk for type 1 diabetes. PLoS One 201 8;13:e0191067.

23. Esteller M. Non-coding RNAs in human disease. Nat Rev Genet 2011;12:861-874

24. Fan B, Luk A, Chan J, Ching R. Micro RNA and Diabetic Complications: A clinical Perspective. Antioxid Redox Signal 2018;29:1041-1063. Epub 2017 Dec 1

25. Pezzolesi MG, Satake E, McDonnell KP, Major M, Smiles AM, Krolewski AS. Circulating TGF- $\beta 1$-Regulated miRNAs and the Risk of Rapid Progression to ESRD in Type 1 Diabetes. Diabetes 2015;64:3285-3293. Epub 2015 Apr 30

26. García-Díaz DF, Pizarro C, Camacho-Guillén P, Codner E, Soto N, Pérez-Bravo F. Expression of miR-155, miR-146a, and miR-326 in T1D patients from Chile: relationship with autoimmunity and inflammatory markers. Arch Endocrinol Metab 2018;62:34-40.

27. van de Bunt M, Gaulton KJ, Parts L, Moran I, R Johnson P, M Lindgren C, Ferrer J, L Gloyn A, I McCarthy M. The miRNA profile of human pancreatic islets and beta-cells and relationship to type 2 diabetes pathogenesis. PLoS One 2013;8:e55272. Epub 2013 Jan 25

28. Marchand L, Jalabert A, Meugnier E, Vanden K, Fabien N, Nicolino M, Madec AM, Thivolet C, Rome S. miRNA-375 a Sensor of Glucotoxicity Is Altered in the Serum of Children with Newly Diagnosed Type 1 Diabetes. J Diabetes Res 2016;2016:1869082. Epub 2016 May 24

29. Pan W, Zhu S, Yuan M, Cui H, Wang L, Luo X, Li J, Zhou H, Tang Y, Shen N. MicroRNA-21 and microRNA-148a contribute to DNA hypomethylation in lupus $\mathrm{CD} 4+\mathrm{T}$ cells by directly and indirectly targeting DNA methyltransferase 1. J Immunol 2010;184:6773-6781. Epub 2010 May 17

30. Osipova J, Fischer DC, Dangwal S, Volkmann I, Widera C, Schwarz K, Lorenzen JM, Schreiver C, Jacoby U, Heimhalt M, Thum T, Haffner D. Diabetes-associated microRNAs in pediatric patients with type 1 diabetes mellitus: a cross-sectional cohort study. J Clin Endocrinol Metab 2014;99:E1661-1665. Epub 2014 Jun 17

31. Backe MB, Novotny GW, Christensen DP, Grunnet LG, Mandrup-Poulsen T. Altering $\beta$-cell number through stable alteration of miR-21 and miR34a expression. Islets 2014;6:e27754.

32. Sheedy FJ, Palsson-McDermott E, Hennessy EJ, Martin C, O'Leary JJ, Ruan Q, Johnson DS, Chen Y, O'Neill LA. Negative regulation of TLR4 
via targeting of the proinflammatory tumor suppressor PDCD4 by the microRNA miR-21. Nat Immunol 2010;11:141-147. Epub 2009 Nov 29

33. Ventriglia G, Nigi L, Sebastiani G, Dotta F. MicroRNAs: Novel Players in the Dialogue between Pancreatic Islets and Immune System in Autoimmune Diabetes. Biomed Res Int 2015;2015:749734. Epub 2015 Aug 3

34. Santos AS, Cunha Neto E, Fukui RT, Ferreira LRP, Silva MER. Increased Expression of Circulating microRNA 101-3p in Type 1 Diabetes Patients: New Insights Into miRNA-Regulated Pathophysiological Pathways for Type 1 Diabetes. Front Immunol 2019;10:1-10.

35. Tian C, Ouyang X, Lv Q, Zhang Y, Xie W. Cross-talks between microRNAs and mRNAs in pancreatic tissues of streptozotocin-induced type 1 diabetic mice. Biomed Rep 2015;3:333-342. Epub 2015 Feb 12

36. Erener S, Marwaha A, Tan R, Panagiotopoulos C, Kieffer TJ. Profiling of circulating microRNAs in children with recent onset of type 1 diabetes. JCI Insight 2017;2:e89656.

37. Wang H, Peng W, Shen X, Huang Y, Ouyang X, Dai Y. Circulating levels of inflammation-associated miR-155 and endothelial-enriched miR-126 in patients with end-stage renal disease. Braz J Med Biol Res 2012;45:1308-1314. Epub 2012 Oct 16

38. Harris TA, Yamakuchi M, Ferlito M, Mendell JT, Lowenstein CJ MicroRNA-126 regulates endothelial expression of vascular cell adhesion molecule 1. Proc Natl Acad Sci U S A 2008;105:1516-1521. Epub 2008 Jan 28

39. Jiang Y, Wang HY, Li Y, Guo SH, Zhang L, Cai JH. Peripheral blood miRNAs as a biomarker for chronic cardiovascular diseases. Sci Rep 2014;4:5026.

40. Bartel DP. MicroRNAs: target recognition and regulatory functions. Cell 2009;136:215-233.
41. Zampetaki A, Kiechl S, Drozdov I, Willeit P, Mayr U, Prokopi M, Mayr A, Weger S, Oberhollenzer F, Bonora E, Shah A, Willeit J, Mayr M. Plasma microRNA profiling reveals loss of endothelial miR-126 and other microRNAs in type 2 diabetes. Circ Res 2010;107:810-817. Epub 2010 Jul 22

42. Bijkerk R, Duijs JM, Khairoun M, Ter Horst CJ, van der Pol P, Mallat MJ, Rotmans JI, de Vries AP, de Koning EJ, de Fijter JW, Rabelink TJ, Zonneveld AJ, Reinders MEJ. Circulating microRNAs associate with diabetic nephropathy and systemic microvascular damage and normalize after simultaneous pancreas-kidney transplantation. Am J Transplant 2015;15:1081-1090. Epub 2015 Feb 25

43. Barutta F, Bruno G, Matullo G, Chaturvedi N, Grimaldi S, Schalkwijk C, Stehouwer CD, Fuller JH, Gruden G. MicroRNA-126 and micro-1 macrovascular complications of type 1 diabetes in the EURODIAB Prospective Complications Study. Acta Diabetol 2017;54:133-139. Epub 2016 Oct 1

44. Satake E, G. Pezzolesi M, Md Dom Z, M. Smiles A, A. Niewczas M, S. Krolewski A. Circulating miRNA Profiles Associated With Hyperglycemia in Patients With Type 1 Diabetes. Diabetes 2018;67:1013-1023. Epub 2018 Feb 16

45. Kassem S, Ariel I, Thornton P, Scheimberg I, Glaser B. Beta-cell proliferation and apoptosis in the developing normal human pancreas and in hyperinsulinism of infancy. Diabetes 2000;49:1325-1333.

46. Tugay K, Guay C, Marques A, Allagnat F, Locke J, Harries LW, A Rutter $G$, Regazzi R. Role of microRNAs in the age-associated decline of pancreatic beta cell function in rat islets. Diabetologia 2016;59:161169.

47. Lu LF, Boldin MP, Chaudhry A, Lin LL, Taganov KD, Hanada T, Yoshimura A, Baltimore D, Rudensky AY. Function of miR-146a in controlling Treg cell-mediated regulation of Th1 responses. Cell 2010;142:914-929. 